Preprint typeset using $\mathrm{LAT}_{\mathrm{E}} \mathrm{X}$ style emulateapj v. 11/10/09

\title{
THE PLATEAU OF GAMMA-RAY BURST: HINT FOR THE SOLIDIFICATION OF QUARK MATTER?
}

\author{
Shi DAI ${ }^{1}$, LiXIN Li $^{2}$, RenXin XU ${ }^{1}$
}

\begin{abstract}
The origin of the shallow decay segment in gamma-ray burst's (GRB) early light curves remains a mystery, especially those cases with a long-lived plateau followed by an abrupt falloff. In this paper, we propose a mechanism to understand the origin of the abrupt falloff after plateau by considering solidification of newborn quark stars with latent heat released as energy injection to GRB afterglow. We estimate the total latent heat released during the phase transition of quark stars from liquid to solid states, to be order of $\sim 10^{51} \mathrm{ergs}$, which is comparable to the emission energy in the shallow decay segment. We also estimate the time scale of radiating the latent heat through thermal photon emission, and find that the time scale agrees with observations. Based on our estimation, we analyze the process of energy injection to GRB afterglow. We show that the steady latent heat of quark star phase transition would continuously inject into GRB afterglow in a form similar to that of a Poyntingflux-dominated outflow and naturally produce the shallow decay phase and the abrupt falloff after plateau. We conclude that the latent heat of quark star phase transition would be an important contribution to the shallow decay radiation in GRB afterglow, and would explain the general features of GRB light curves (including the plateau), if pulsar-like stars are really (solid) quark stars.
\end{abstract}

Subject headings: $\gamma$-rays: bursts, X-rays, neutron stars, elementary particles

\section{INTRODUCTION}

NASA's broadband (gamma-ray, X-ray, UV \& optical) Swift satellite's (Gehrels et al. 2004) successful launch and operation opens a brand new era in the observation of the gamma-ray burst (GRB) phenomenon and has revolutionized our understanding of GRBs in many aspects (Mészáros 2006; Zhang 2007). The prompt slewing capability of Swift allows us to swiftly catch the very early signals following the GRB prompt emission and the precise localizations make it possible for ground-based follow up observations of most bursts. A large number of wellsampled X-ray light curves from tens of seconds to days past the GRB triggers (Burrows et al. 2004; Liang et al. 2007) have been accumulated through Swift's observation, which provide us a good chance to investigate early afterglow and study GRB systematically.

A canonical light curve of X-ray afterglows as revealed by Swift (Zhang et al. 2006; Nousek et al. 2006; OBrien et al. 2006) is composed of five parts: prompt gammaray phase with a tail, shallow decay phase, normal decay phase, jetlike decay phase, and erratic X-ray flares. The physical origins of these segments have been widely discussed in the literature (Zhang et al. 2006; Nousek et al. 2006; Dai et al. 2006; Liang et al. 2007). In this paper, we mainly focus on the shallow decay phase and the subsequent phase. On the one hand, the physical origin of the shallow decay phase is still a mystery. Different models, such as the energy injection models (Zhang et al. 2006; Nousek et al. 2006; Panaitescu et al. 2006b), a combination of the GRB tail with delayed onset of the afterglow emission (Kobayashi \& Zhang 2007), off-beam jets (Toma et al. 2006; Eichler \& Granot 2006), precursor activity (Ioka et al. 2006), two-component jets (Gra-

\{daishi, lxl, r.x.xu\}@pku.edu.cn

${ }^{1}$ School of Physics and State Key Laboratory of Nuclear Physics and Technology, Peking University, Beijing 100871, China;

${ }^{2}$ Kavli Institute for Astronomy and Astrophysics, Peking University, Beijing 100871, China. not et al. 2006; Jin et al. 2007), two-component emission model (Yamazaki 2009), varying microphysical parameters (Ioka et al. 2006; Panaitescu et al. 2006a; Fan \& Piran 2006; Granot et al. 2006) and so on, are hard to differentiate among each other from the X-ray observations (Zhang 2007). On the other hand, some puzzling facts related to the shallow decay phase are revealed by Swift's observations, which can not be explained by current models. Two interesting facts are that the break between the shallow and the normal decay segments in the X-ray light curve for some GRBs is chromatic (Panaitescu et al. 2006a; Fan \& Piran 2006) and that the light curve of GRBs like GRB 070110 shows a long-lived plateau followed by an abrupt falloff (the decay slope is about -9 , with time zero at the trigger) (Liang et al. 2007). The former fact suggests that the optical and Xray emission in the shallow decay phase may not be the same component, and the later fact indicates an internal origin of the X-ray plateaus and continuous operation of a long-term central engine. Systematically analysis of the Swift X-Ray Telescope data suggests that the physical origin of the shallow decay phase is diverse (Liang et al. 2007) and we expect to get more information from it including the physics of dense material and possible gravitational wave (Alessandra \& Mészáros 2009).

We note that GRB central engine may relate to the physics of cold matter at supra-nuclear density, which is now one of the daunting challenges in particle physics. Cold quark matter is conjectured to be in a solid state at realistic baryon densities of compact stars (e.g., $\mathrm{Xu}$ 2010 , for a review), we are then considering latent heat of quark star phase transition from liquid to solid as energy injection to GRB afterglow in order to understand the feature of plateau followed by an abrupt falloff in some GRB's light curve. Quark stars, as possible nature of pulsars, are likely to form in GRB, no matter in the process of high-mass star collapses or merge of binary neutron stars. In the solid quark star model, which is suc- 
cessful to understand a variety of pulsars' observational features ( $\mathrm{Xu} 2003$ ), it is likely that as the temperature of quark star drops after GRB, a phase transition from liquid to solid would happen (Xu \& Liang 2009). Since the quark star in phase transition would emit energy with constant temperature and solid quark star would cool very fast due to its low heat capacity (Yu \& Xu 2009), the latent heat of this quark star phase transition not only provides a long-term steady central engine, but also naturally shows a abrupt cutoff when the phase transition ends. In this paper, we show that not only the energy released during the phase transition and the time scale of radiating latent heat agree with observations, but the process of energy injection to GRB afterglow is also reasonable. Thus latent heat of quark star phase transition as energy injection to GRB afterglow would be an prospective model to understand the physical origin of the shallow decay phase of GRB light curve, especially for the feature of abrupt falloff after plateau.

In Section 2, we estimate the latent heat of quark star phase transition in solid quark star model. Section 3 discusses the process of energy injection. We conclude the results in Section 4.

\section{LATENT HEAT OF QUARK STAR PHASE TRANSITION}

To estimate the latent heat of quark star phase transition from liquid to solid, we need to know the state of cold quark matter and the interaction between quarks. However, due to the non-perturbative effect of the strong interaction between quarks at low energy scale and the many-body problem of vast assemblies of interacting particles, we can not describe the state of cold quark matter from first principle up to now. Yet, it is phenomenologically conjectured that astrophysical cold quark matter could be in a solid state, and a variety of observational features, which may challenge us in the hadron star model, could be naturally understood in the solid quark star model (Xu 2003). Recent results of relativistic heavy ion collision experiments also show that the interaction between quarks is very strong in hot quarkgluon plasma (Shuryak 2009), then it is reasonable to conjecture that the interaction between quarks should be stronger in cold quark matter. The strong interaction may then make quarks grouped in clusters, and if the residual interaction between quark clusters is stronger than their kinetic energy, each quark cluster could be trapped in the potential well and cold quark matter will be in a solid state (Xu 2010).

Considering that a single quark cluster inside a quark star is assumed to be colorless, just like each molecule in a bulk of inert gas is electric neutral, Lai and $\mathrm{Xu}$ in their recent work (Lai \& Xu 2009) used Lennard-Jones potential to describe the interaction between quark clusters in quark stars and got the equation of state of quark stars. The interaction is expressed as

$$
u(r)=4 U_{0}\left[\left(\frac{r_{0}}{r}\right)^{12}-\left(\frac{r_{0}}{r}\right)^{6}\right],
$$

where $U_{0}$ is the depth of the potential and $r_{0}$ can be considered as the range of interaction.

Based on this solid quark star model, we can then estimate the latent heat of quark star phase transition from liquid to solid. Rather than performing difficult molecular dynamics simulations of crystallization, we would prefer estimating the latent heat in order of magnitude by analogy with inert gas and common substances since the quark clusters are non-relativistic and the interaction is similar to common substances. In Table1 below, we list the melting heat, heat of vaporization, potential and ratio of melting heat to potential of inert gas and some common substances. The melting heat corresponds to the latent heat, and for substances with known heat of sublimation, we equal the potential to heat of sublimation, otherwise we equal the potential to the sum of melting heat and heat of vaporization.

From the data, we can see that for most substances the ratio of melting heat to potential is between 0.1 to 0.01 . Considering that the interaction between quark clusters is similar to inert gas and is relatively strong, we choose the ratio of potential to melting heat to be $f \approx 0.1 \sim$ 0.01 for estimation. Then based on the solid quark star model proposed by Lai, choosing $U_{0}=100 \mathrm{MeV}$ (Lai \& $\mathrm{Xu} 2009$ ), we can estimate the energy released by each quark cluster in the liquid to solid phase transition as

$$
E_{\text {cluster }} \sim f U_{0} \approx 1 \sim 10 \mathrm{MeV} .
$$

For quark star of one solar mass, $M_{\odot} \approx 2 \times 10^{33} \mathrm{~g}$, the number of baryon is $n=10^{57}$, then the total energy released during the phase transition can be estimated as

$$
E=E_{\text {cluster }} n \approx 10^{51} \sim 10^{52} \text { ergs. }
$$

This order of magnitude agrees with the typical energy released in the shallow decay phase of GRB, that is to say, the latent heat of quark star phase transition from liquid to solid is sufficient to produce the plateau.

On the other hand, according to the Lindemann law that a solid melts when the root-mean-square amplitude of atomic vibrations exceeds a certain fraction of the equilibrium nearest neighbor distance, we can estimate the temperature of quark star phase transition and further more the time scale of radiating latent heat. In Mohazzabi and Behroozi's work in 1987, they obtained the expression of the ratio of the root-mean-square amplitude of atomic vibrations to the equilibrium nearest neighbor distance for inert gas, and found that the Lindemann law being well consistent with experiments (Mohazzabi \& Behroozi 1987). As for our estimation, we can consult the ratio of potential to heat, $\Gamma=U_{0} / k T$, for common substances, and then get the temperature of quark star phase transition by analogy. For onecomponent plasma, $\Gamma \approx 175$ (DeWitt et al. 2001), for multi-component plasma, $\Gamma \approx 233$ (Horowitz et al. 2007). So choosing $\Gamma \approx 200, U_{0}=100 \mathrm{MeV}$, the temperature of quark star phase transition is around $0.5 \mathrm{MeV}$.

During the liquid to solid phase transition, the temperature of quark star would remain constant, and the latent heat would be released through thermal emission. Then the time scale of radiation can be estimated as

$$
t=\frac{E}{\sigma T^{4} 4 \pi R^{2}}
$$

where, $E=10^{51}$ ergs, $\sigma$ is the Stefan-Boltzman constant, $R=10 \mathrm{~km}$ is the radius of quark star, and $T \approx 0.5 \mathrm{MeV}$, we find that the time scale of radiation is $t \approx 1000 \mathrm{~s}$, which agrees with observations of GRB afterglow plateau.

After solidification, since the heat capacity of solid 
quark stars is very low ( $\mathrm{Yu} \& \mathrm{Xu} 2009$ ), the central quark star would cool rapidly. The residual inner energy would be released almost immediately, thus an abrupt cutoff of energy injection to the afterglow would appear, and naturally result in an abrupt falloff after the shallow decay phase. In Fig1, a schematic cooling curve of quark star is presented, which consists of three stages. The first stage could be an initial fast cooling stage due to the emission of neutrinos and photons at the very beginning of a quark star, when its initial temperature $T_{0}$ could be much higher than $10 \mathrm{MeV}$. When the temperature of quark star drops to the melting point $T_{p}$, it could come to the second stage, the liquid to solid phase transition which last from $t_{i}$ to $t_{f}$. At this stage, the temperature of quark star would remain constant and the latent heat would be released steadily which could provide a longlived steady central engine. After phase transition, the born solid quark star would rapidly release its residual inner energy due to its low heat capacity, thus a steep falloff appears in the cooling curve corresponding to the abrupt falloff after the shallow decay segment.

\section{THE STANDARD FIREBALL REVISITED?}

In the standard GRB fireball model, it is assumed that a large amount of energy is instantly released through some explosion process. The energy drives some material to expand with a ultra-relativistic speed, which requires that the fireball outflow is low-baryon-loaded so that the total rest mass of the fireball is sufficiently small. The standard fireball is assumed to be highly nonuniform and in the extreme case is composed of many distinct shells. Collision of different shells produces internal shock waves, which are thought to be responsible for the observed prompt emission. Collision of shells with the surrounding intermediate stellar material produces external shock waves, which are thought to be responsible for the observed GRB afterglow emission. While for the afterglow flares, they are usually thought to be produced by later injection of energy through internal shock.

After having estimated the energy released by quark star phase transition and the time scale of radiation, it is necessary for us to qualitatively discuss the process of energy injection to GRB afterglow. Generally speaking, the energy injection to afterglow could consist of some kinetic-energy (i.e., baryons) dominated shells or a Poynting-flux-dominated wind (Usov 1994; Mészáros \& Rees 1997). In case of afterglow, we always consider an impulsive shell that is already heated during the shell-ISM interaction and that is collecting material from the ISM, and in the meantime also receives a large enough injection energy from a continuous Poynting-fluxdominated wind or a kinetic-energy dominated shell. As discussed in literatures (Zhang \& Mészáros 2001, 2002), for Poynting-flux-dominated wind case in which pure energy with negligible baryon loading is injected to fireball, no reverse shock is expected, and the injection signature is produced only by the forward shock emission. For kinetic-energy-dominated matter shells case, depending on whether the collision between the injected and the impulsive shell is mild or violent, the injection process is quite different. If the relative velocity between the colliding shells does not exceed a critical value defined by their energy ratio, the collision is mild, and the injection may be analogous to the Poynting-flux injection case. Other- wise, the injection is violent, and an additional pair of strong shocks will form at the discontinuity between two colliding shells which will greatly influence the injection signature.

As for our energy injection model, the latent heat released during quark star phase transition would produce a radiation-dominated fireball, whose luminosity is about $L \approx 10^{48} \mathrm{ergs} / \mathrm{s}$ according to our estimation. The optical depth can then be estimated as

$$
\begin{gathered}
\tau_{\gamma \gamma}=\frac{f_{p} \sigma_{T} F D^{2}}{R^{2} m_{e} c^{2}} \\
=10^{13} f_{p}\left(\frac{D}{3000 \mathrm{Mpc}}\right)^{2}\left(\frac{F}{10^{-11} \mathrm{ergs} / \mathrm{cm}^{2}}\right)\left(\frac{R}{10 \mathrm{~km}}\right)^{-2},
\end{gathered}
$$

where, $R=10 \mathrm{~km}$ is the radius of quark star. The optical depth is very large, so the energy flux from central quark star would form a pure radiation fireball consist of radiation and electron-positron pairs, which is similar to the fireball of prompt emission but continuous instead. We expect that this pure radiation fireball would expand and finally inject energy to the afterglow in form of Poynting flux. If we consider possible baryons in the space the fireball swept, we can estimate the velocity the matter shell could reach assuming that all the energy are converted to the kinetic energy of the baryons. Since in this late injection phase the baryon loading could be in principle much lower, we assume that the density of baryons are $10 \%$ of the original density in space. Then we can estimate as

$$
\gamma=E / M c^{2}
$$

For $E \approx 10^{51}$ ergs, we get $\gamma \approx 100$. According to Zhang and Mészáros' work in 2002 (Zhang \& Mészáros 2002), for such kinetic-energy-dominated matter shells with $\gamma \approx 100$ and $L \approx 10^{48} \mathrm{ergs} / \mathrm{s}$, the collision between the injected and the impulsive shell is mild, and the injection may be analogous to the Poynting-flux injection case.

\section{CONCLUSIONS}

A possible physical origin of the shallow decay phase of GRB light curve is proposed in the solid quark star model. We suggest that quark stars may be born in GRB, and as the temperature of quark star drops, a phase transition from liquid to solid may occur in the quark star. The latent heat of phase transition would provide a long-lived steady energy injection to GRB afterglow, since the temperature of central star would remain constant during phase transition. When phase transition ends, an abrupt falloff after the plateau in the light curve would naturally appear which is hard to understand by other central engine models.

We estimate the latent heat of quark star phase transition from liquid to solid based on the solid quark star model whose interaction between quark clusters is described by Lennard-Jones potential. The energy of $\sim 10^{51}$ ergs and the radiation time scale of $\sim 10^{3}$ seconds agree with observations of the shallow decay phase. We also qualitatively discuss the process of energy injection to afterglow, and show that the energy injection of phase transition would be in a form similar to that of the Poynting-flux-dominated outflow. Both the estimation and the injection process suggest that the idea of 
considering latent heat of quark star phase transition as energy injection to GRB afterglow is rational.

We would like to acknowledge useful discussions at our pulsar group of PKU. This work is supported by the Na- tional Natural Science Foundation of China (10973002, 10935001), the National Basic Research Program of China (2009CB824800), and the National Fund for Fostering Talents of Basic Science (J0630311).

\section{REFERENCES}

Alessandra, C., \& Mészáros, P. 2009, ApJ, 702, 1171

Burrows, D. N., et al. 2004, Proc. SPIE, 5165, 201

Dai, Z. G., Wang, X. Y., Wu, X. F., Zhang, B. 2006, Science, 311, 1127

Dean, J. A. 1999, Lange's Chemistry Handbook (Version 15th, in Chinese), Table 6.2.

DeWitt, H., et al. 2001, Contrib Plasma Phys, 41, 251

Eichler, D., \& Granot, J. 2006, ApJ, 641, L5

Fan, Y., \& Piran, T. 2006, MNRAS, 369, 197

Gehrels, N. et al. 2004, ApJ, 611, 1005.

Granot, J., Königl, A., \& Piran, T. 2006, MNRAS, 370, 1946

Horowitz, C. J., et al. 2007, Physical Review E, 75, 066101

Ioka, K., Toma, K., Yamazaki, R., \& Nakamura, T. 2006, A\&A, 458,7

Jin, Z.-P., Yan, T., Fan, Y.-Z., \& Wei, D.-M. 2007, ApJ, 656, L57

Kobayashi, S., \& Zhang, B. 2007, ApJ, 655, 973

Lai, X. Y., \& Xu, R. X. 2009, MNRAS, 398, 31

Liang, E. W., Zhang, B. B., Zhang, B. 2007, ApJ, 670,565

Lindemann, F. A. 1910, Physik Z., 11, 609

Mészáros, P. 2006, Rep. Prog. Phys., 69, 2259

Mészáros, P., \& Rees,M. J. 1997, ApJ, 482, 29

Mohazzabi, P. \& Behroozi, F. 1987, Journal of Materials Science Letters, 6, 404
Nousek, J. A., et al. 2006, ApJ, 642, 389

OBrien, P. T., et al. 2006, ApJ, 647, 1213

Panaitescu, A., Mészáros, P., Burrows, D., Nousek, J., Gehrels, N., OBrien, P., \& Willingale, R. 2006a, MNRAS, 369, 2059

Panaitescu, A., Mészáros, P., Gehrels, N., Burrows, D., \& Nousek, J. 2006b, MNRAS, 366, 1357

Shuryak E. V., 2009, Prog. Part Nucl. Phys., 62, 48

Toma, K., Ioka, K., Yamazaki, R., \& Nakamura, T. 2006, ApJ, 640,139

Usov, V. V. 1994, MNRAS, 267, 1035

$\mathrm{Xu}$, R. X. Solid quark stars. 2003, ApJ, 596, 59

Xu R. X., 2010, IJMP D (arXiv:1002.4469, in press)

Xu, R. X., \& Liang, E. W. 2009, Sci China Ser G-Phys Mech Astron, 52, 315

Yamazaki, R. 2009, ApJ, 690, 118

Yu, M., \& Xu, R. X. 2009, arXiv:0905.3818

Zhang, B. 2007, Chinese J. Astron. Astrophys., 7, 1

Zhang, B., \& Mészáros, P. 2001, ApJ, 552, 35

Zhang, B., \& Mészáros, P. 2002, ApJ, 566, 712

Zhang, B., Fan, Y.-Z., Dyks, J., Kobayashi, S., Mészáros, P., Burrows, D. N., Nousek, J. A., \& Gehrels, N. 2006, ApJ, 642, 354 
FIG. 1.- A schematic cooling behavior of a new-born quark star. Three stages are shown: an initial cooling stage due to the emission of neutrinos and photons at the very beginning of a quark star with initial temperature $T_{0}$, a liquid to solid phase transition stage from time $t_{i}$ to $t_{f}$ with constant temperature $T_{p}$, and, after solidification, a fast cooling stage because of solid quark star's low heat capacity. We focus on the duration from $t_{i}$ to $t_{f}$ in this paper.

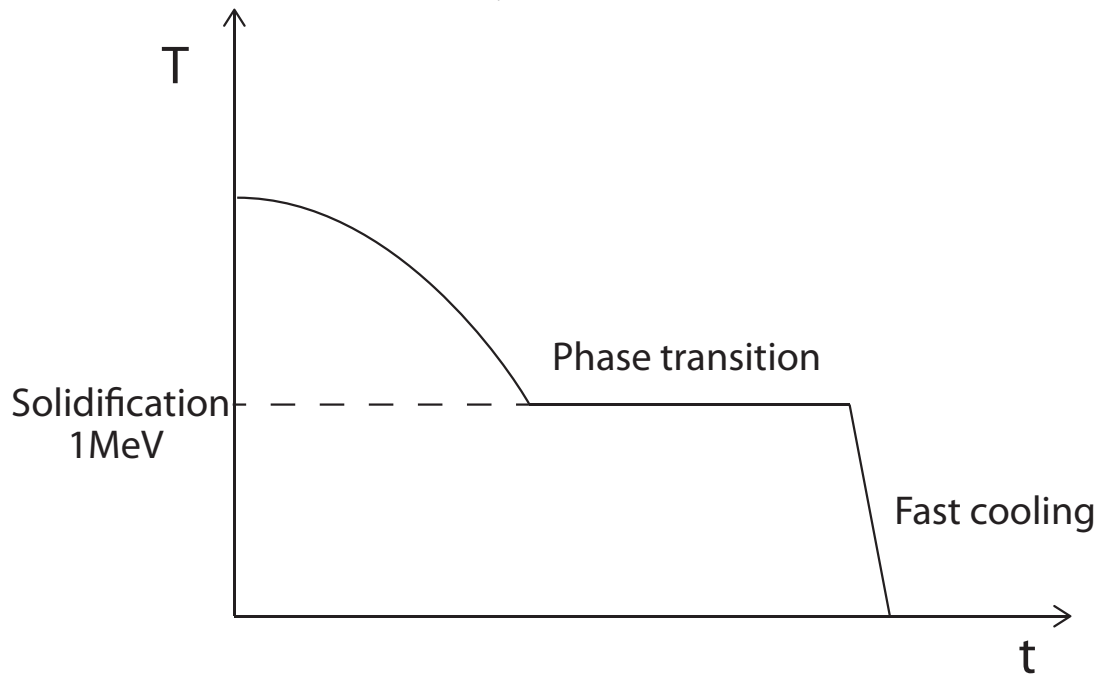

TABLE 1

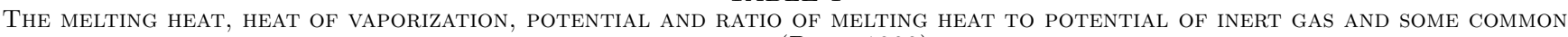
SUBSTANCES (DEAN 1999).

\begin{tabular}{|c|c|c|c|c|}
\hline & melting heat kcal/mol & heat of vaporization kcal/mol & potential kcal/mol & melting heat/potential \\
\hline $\mathrm{He}$ & 0.0033 & 0.0194 & 2.2944 & 0.0014 \\
\hline $\mathrm{Ne}$ & 0.0801 & 0.422 & 9.1776 & 0.0087 \\
\hline $\mathrm{Xe}$ & 0.5495 & 3.02 & 12.6192 & 0.0436 \\
\hline $\mathrm{Rn}$ & 0.69 & 4.01 & 19.5024 & 0.0354 \\
\hline & & & & 78 \\
\hline $\mathrm{Al}$ & 2.56 & 69.5 & 18.3 & 0.0328 \\
\hline $\mathrm{Cs}$ & 0.499 & 16.198 & 81 & 0.0272 \\
\hline $\mathrm{Cu}$ & 3.17 & 72.74 & 99.5 & 0.0391 \\
\hline $\mathrm{Fe}$ & 3.63 & 83.68 & 14.65 & 0.0365 \\
\hline $\mathrm{Hg}$ & 0.5486 & 14.13 & 25.75 & 0.0375 \\
\hline $\mathrm{Na}$ & 0.622 & 23.285 & 107.7 & 0.0242 \\
\hline $\mathrm{Si}$ & 12 & 85.8 & 171.29 & 0.1114 \\
\hline $\mathrm{C}$ & 25 & & 1.644 & 0.1460 \\
\hline $\mathrm{CO}$ & 0.2 & 1.444 & 6.03 & 0.1217 \\
\hline $\mathrm{CO}{ }_{2}$ & 1.99 & & 11.153 & 0.3300 \\
\hline $\mathrm{H}_{2} \mathrm{O}$ & 1.436 & 9.717 & 12.34 & 0.1287 \\
\hline $\mathrm{H}_{2} \mathrm{O}_{2}$ & 2.987 & 10.53 & 77.5 & 0.2421 \\
\hline $\mathrm{CaCl}_{2}$ & 6.8 & 56.2 & & 0.0877 \\
\hline
\end{tabular}

